\title{
Field test of a new snow-particle counter (SPG) system
}

\author{
TAKeshi SATO, TADASHI Kimura, \\ Shinjo Branch of Snow and Ice Studies, National Research Institute for Earth Science and Disaster Prevention, \\ Shinjo, Yamagata 996, Japan \\ TAMinoe Ishimaru AND TOSHISUke MARUyama \\ Niigata Electric Company Ltd., Sawada, Nagaoka, Niigata 940-11, Japan
}

\begin{abstract}
The optical system of the snow-particle counter (SPC), which was developed by Schmidt in 1977, has been improved. A laser diode is used as a light source, achieving uniform sensitivity to a blowing snow particle regardless of the location of particle trajectory within a sampling volume. The light entering a slit, which may be affected by a blowing snow particle, is perfectly detected by use of a plano-cylindrical lens and a dual-type photodiode. A signal processor has been developed to get output voltage proportional to the mass flux of blowing snow.

From the estimates based on blowing snow characteristics and wind speed profile, the new SPC system can accurately detect all the particles of effective sizes at least at a height above $0.1 \mathrm{~m}$ when the wind speed at a height of $1 \mathrm{~m}$ is less than $15 \mathrm{~m} \mathrm{~s}^{-1}$.

Considering the Fraunhofer diffraction by both the wire and the particle, the relation between a particle diameter and sensor output of the new SPC system is derived from the calibration with spinning wires.

Mass flux obtained with the new SPC system was found to be close to that with a snow trap. The system was operated continuously for at least nine days using two $35 \mathrm{~A}$ h lead batteries.
\end{abstract}

\section{INTRODUCTION}

Observations of blowing snow have been carried out with various types of snow traps and photoelectric instruments. Manual operations, such as weighing the collected snow, are necessary with snow traps, which renders them unsuitable for continuous measurements of blowing snow. In contrast, photoelectric instruments can continuously detect blowing snow particles passing through a light beam and their electric signal processing enables an automatic recording of blowing snow.

The photoelectric instrument was first proposed during the 1960s. Schmidt (1977) developed an instrument for practical use, the so-called snow-particle counter (SPC), which measures the particle size, frequency and speed. After that, Gubler (1981) proposed an instrument to monitor the mass flux of blowing snow remotely for a long period, in which mass flux is obtained by electric signal processing. Brown and Pomeroy (1989) developed a particle detector which only counts the particles breaking a light beam. Mass flux is calculated from the count of particles under the assumption of typical size distributions for the saltation and suspension layers.

The authors have improved the optical system of the SPC developed originally by Schmidt and have designed a signal processor whose output is directly proportional to the mass flux of blowing snow. The present paper describes the optical system and signal processor of the new SPC system and discusses accuracy of the SPC measurement and problems related to calibration. Comparisons with a snow trap are also shown.

\section{OPTICAL SYSTEM}

The sensor consists of the light source and receiver as shown in Figures 1 and 2. The light source in a tube is

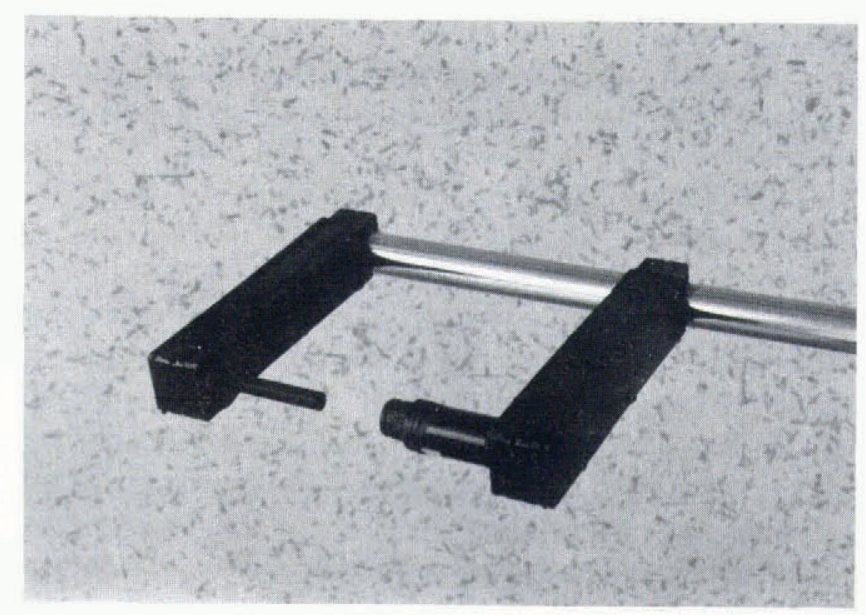

Fig. 1. Appearance of new SPC sensor. Light source in a tube is attached to the enclosure on the righthand side; and light receiver with a thin hood is attached to the enclosure on the lefthand side. 


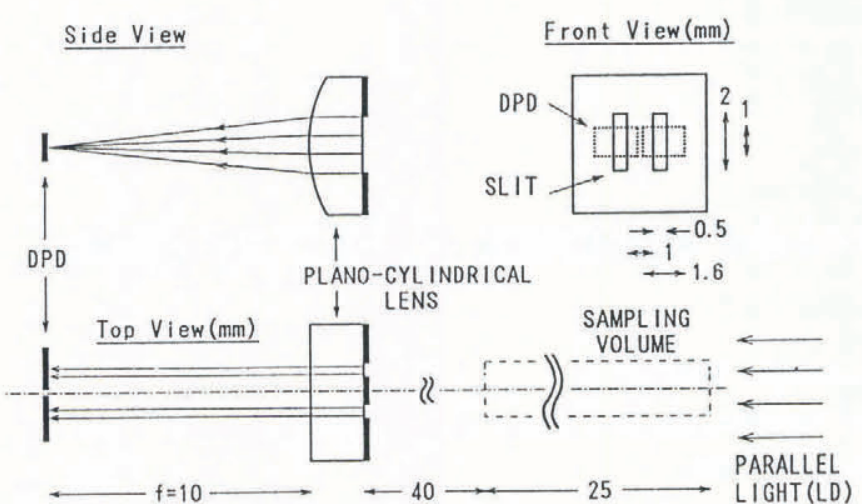

Fig. 2. Schematic of the light receiver.

attached to the enclosure on the righthand side and a glass disk covers the open end of the tube. This enclosure contains a driver circuit for light emission. The light receiver is attached to another enclosure containing a preamplifier. A hood of $40 \mathrm{~mm}$ length and $4 \mathrm{~mm}$ inner diameter is fixed to the light receiver. This hood is used so that a sampling volume does not suffer from aerodynamic interference by the enclosure. The open end of the hood is also covered with a glass disk. The light path between the end of the light source and the end of the hood is $25 \mathrm{~mm}$ long.

The new SPC system adopts a double-slit type sensor so that external light does not affect the measurement. Each slit is $0.5 \mathrm{~mm}$ wide and $2 \mathrm{~mm}$ long and the two slits are $1 \mathrm{~mm}$ apart. Except for the slit width, these dimensions are smaller than those of the Schmidt's sensor. The sampling volume defined by Schmidt (1977) is $100 \mathrm{~mm}^{3}$ ( $25 \mathrm{~mm}$ wide, $2 \mathrm{~mm}$ high and $2 \mathrm{~mm}$ deep).

A laser diode (LD) with a collimator is used as a light source, with $780 \mathrm{~nm}$ wavelength. Since the emitting part of the $\mathrm{LD}$ ( $1 \mu \mathrm{m}$ wide and $0.5 \mu \mathrm{m}$ high) is much smaller than blowing snow particles, the collimated light beam of $8 \mathrm{~mm}$ diameter is considered to be parallel. This ensures that the sensitivity to a particle does not depend on the location of the particle trajectory within the sampling volume, which has been confirmed experimentally (Kimura, 1991).

A dual-type photodiode (DPD) detects interruption of the light beam by a blowing snow particle. The DPD is placed at a focal point of the plano-cylindrical lens which is attached just behind the double-slits. The size of each receiving element of the DPD $(1.6 \mathrm{~mm}$ wide and $1.0 \mathrm{~mm}$ high) allows detection of all of the light entering each paired slit.

The power required is: $+12 \mathrm{~V}, 60 \mathrm{~mA} ;-12 \mathrm{~V}, 5 \mathrm{~mA}$. Detailed structures and other specifications of the light source and receiver are described in Kimura (1991).

\section{SIGNAL PROCESSOR}

A block diagram of the signal processor is shown in Figure 3. Its original type was developed by Kimura (1991) and the present type includes additional electric circuits.

When a particle passes through the sampling volume, first the light energy entering the upwind slit decreases, and then that entering the downwind slit decreases. Thus, a pair of successive voltage pulses with the same peak magnitude and opposite signs is generated by the DPD and a differential preamplifier. The voltage of these pulses corresponds to the difference in the decrease of detected light energy between the upwind slit and the downwind slit. The gain of the preamplifier is adjusted by considering a particle-diameter range to be detected. The positive part of this pair of pulses is eliminated, and the remaining negative pulse is again amplified and inverted. The peak voltage of the pulse is almost in proportion to the cross-sectional area of the particle.

For mass flux measurements, the voltage pulse is raised by 1.5 by a functional amplifier so that the raised peak voltage becomes proportional to the volume of the particle. Then, the peak voltage of the resultant pulse is held, and the peak hold pulses are integrated every second. The integration is performed by a $\mathrm{V} / \mathrm{F}$ converter and a counter. Final output voltage is given by a D/A converter, which is proportional to the one-second averaged mass flux. Power requirements of the signal processor are: $+12 \mathrm{~V}, 90 \mathrm{~mA} ;-12 \mathrm{~V}, 60 \mathrm{~mA}$.

The signal processor has four mass flux ranges, and a range adequate to the intensity of blowing snow can be selected. The width of the peak hold pulse is changed with a selected mass flux range. The new SPC system includes a data logger, which can record about 30000 voltage measurements averaged over the period from one minute to one hour.

Though the present new SPC system and Gubler's flux

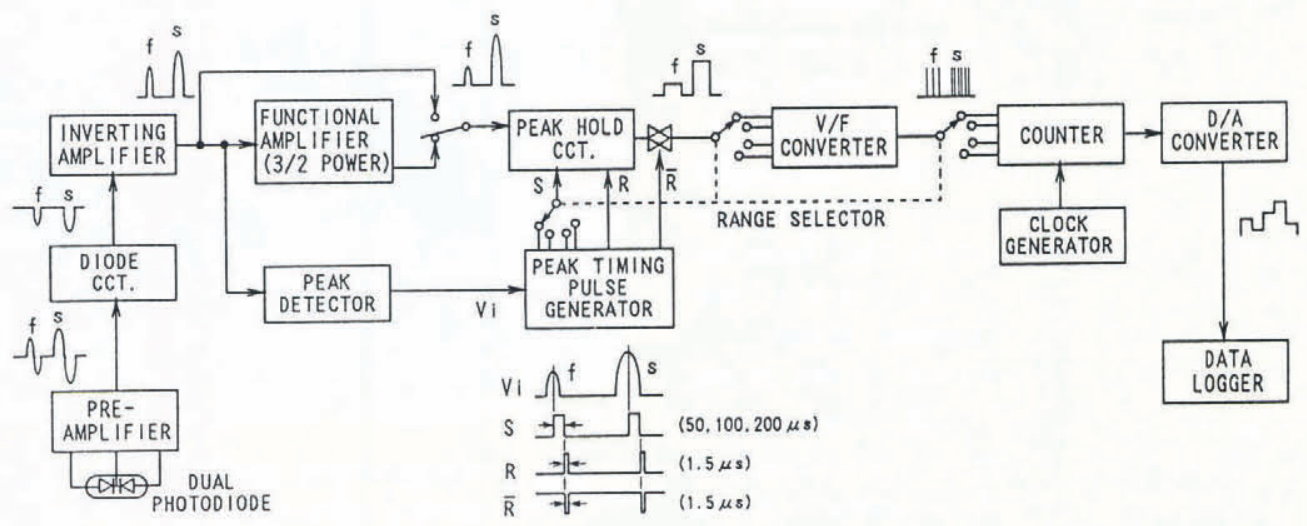

Fig. 3. Block diagram of the signal processor. 
meter (1981) aim at direct measurement of the mass flux of blowing snow, their signal processors operate differently. In Gubler's processor, particle sizes are resolved into five classes. When a particle is detected, a pulse train is generated whose length is proportional to the mean particle volume of the corresponding class. On the other hand, the present processor incorporates a functional amplifier to obtain volume-proportional voltage pulses. In the course of the electric integration of these pulses, particle sizes are resolved into substantially more classes than Gubler's five. The resolved classes vary from 25 to 100 with a selected mass flux range.

\section{ACCURACY OF MEASUREMENT}

The new SPC system is designed for the measurement of blowing snow near a snow surface. For accurate measurement, the system should satisfy at least two requirements: the sensor signal should have a sufficient level within a particle-size range to be measured; and all the particles of effective sizes passing through the sampling volume should be detected accurately. Considering the principles of particle detection and signal processing, the latter requirement is satisfied if more than two particles do not exist simultaneously in the sampling volume, and if the width of the peak hold pulse is less than the time interval for two particles to pass successively.

The accuracy of mass flux measurement depends on blowing snow characteristics as well as specifications of the new SPC system. In this study, particle-size distributions are modeled by the gamma function as

$$
P(d)=\frac{d^{\alpha-1}}{\beta^{\alpha} \Gamma(\alpha)} \exp (-d / \beta),
$$

where $d$ is a particle diameter, and the parameters, $\alpha$ and $\beta$, are summarized in Table 1 . They are determined from the results of direct particle sampling in Antarctica by Budd (1966) and Nishimura and Maeno (1983). These size distributions are assumed to be independent of wind speed, because Budd (1966) did not observe a remarkable change in the mean particle size with wind speeds of 12 to $21 \mathrm{~m} \mathrm{~s}^{-1}$. The snowdrift density profile observed in Antarctica by Budd and others (1966) is used as a function of wind speed and height above a surface.

The volume contributions calculated by Equation (1)

Table 1: Summary of the particle-size distribution

$\begin{array}{cccc}\text { Height } & \alpha & \beta & \text { Mean diameter } \\ \mathrm{m} & & \mu \mathrm{m} & \mu \mathrm{m}\end{array}$

\begin{tabular}{cccc}
0.03 & 10 & 20 & 200 \\
0.1 & 10 & 15 & 150 \\
1 & 15 & 6.7 & 100 \\
10 & 15 & 5.3 & 80 \\
\hline
\end{tabular}

indicate that it is sufficient to detect the particles of diameter $50-300 \mu \mathrm{m}$ for the mass flux measurement at a height above $0.1 \mathrm{~m}$.

On the basis of the log-profile of wind speed together with the particle-size distribution and the snowdrift density profile, number density and number flux of blowing snow particles of effective sizes are calculated. Thus, the latter requirement can be examined as a function of wind speed $\left(10-20 \mathrm{~m} \mathrm{~s}^{-1}\right)$ and elevation $(0.03-$ $10 \mathrm{~m}$ ), while previous researchers discussed it by use of a particle of typical size (e.g., Schmidt, 1977; Gubler, 1981). For simplification, the effect of diffraction by a particle is not considered here, but will be described later.

Results are summarized as follows: when the wind speed at a height of $1 \mathrm{~m}$ is $10 \mathrm{~m} \mathrm{~s}^{-1}$, the number of particles in the sampling volume is less than two at a height above $0.03 \mathrm{~m}$. When the wind speed is $15 \mathrm{~m} \mathrm{~s}^{-1}$, the same holds at a height above $0.1 \mathrm{~m}$. However, when the wind speed is $20 \mathrm{~m} \mathrm{~s}^{-1}$, more than two particles exist simultaneously in the sampling volume at a height less than $5 \mathrm{~m}$, which means that either the sensor signal is affected by other particles or multiple particles are detected as a single particle. Unless the wind speed exceeds $15 \mathrm{~m} \mathrm{~s}^{-1}$, the peak hold pulse width is less than the time interval for two particles to pass successively. When the wind speed is $20 \mathrm{~m} \mathrm{~s}^{-1}$, the requirement for the width of the peak hold pulse is not satisfied at a height below several meters and some of the particles are not detected.

It is thus concluded that all the particles of effective sizes can be detected accurately at least at a height above $0.1 \mathrm{~m}$ if the wind speed is less than $15 \mathrm{~m} \mathrm{~s}^{-1}$. Under stronger wind conditions, the sampling volume and the width of the peak hold pulse should be reduced for accurate mass flux measurement. A single-slit type sensor with smaller sampling volume is preferable from this point of view.

Although the present examination takes account of the particle-size distribution in calculating the number density, it does not consider the difference in the mass flux contribution between the particles of various sizes. If this difference is considered, the observational restriction may be somewhat lightened so far as mass flux values are concerned.

\section{SENSOR GALIBRATION}

Spinning wires of different diameters are used in the calibration of the new SPC sensor (Kimura, 1991). The sensor is positioned so that a wire passes through the center of the sampling volume and moves perpendicular to the light beam. The sensor is then inclined so that the projection of the wire intersects the slit at an angle of $45^{\circ}$. The calibration curve for a blowing snow particle, which is assumed to be a sphere, is obtained from the wirecalibration data following the procedure which is described later.

When a particle is illuminated by parallel light, optical phenomena associated with the particle are described by the geometrical optics if its diameter is much larger than the wavelength of the light; otherwise diffraction by the particle cannot be ignored. The effect of diffraction by one particle on the SPC measurement is discussed below. 
Since diffraction has an angular pattern, some portion of diffracted energy enters the upwind slit (slit 1) and some other portion enters the downwind slit (slit 2). Considering the principle of particle detection of the new SPC system mentioned above, these diffracted lights have reverse effects on the sensor signal. Hereafter, the sensor output $V_{\mathrm{p}}$ denotes the magnitude of the peak output voltage from the preamplifier, which is observed when the projection of the particle is at the center of slit 1 . The principle of particle detection is formulated as

$$
V_{\mathrm{p}} \propto 2 I_{0} A_{\mathrm{p}}-D_{\mathrm{p} 1}+D_{\mathrm{p} 2}
$$

where the suffix $\mathrm{p}$ denotes a particle, $I_{0}$ is the incident energy of parallel light per unit area, and $A_{\mathrm{p}}$ is a geometrical cross-section of the particle. The first term on the righthand side of Equation (2) represents the reduction of incident energy by the particle, which is detected through slit 1 . The factor 2 is the extinction efficiency derived from the classical wave optics (Van de Hulst, 1981). The terms $D_{\mathrm{p} 1}$ and $D_{\mathrm{p} 2}$ are the detected portions of diffracted energy through slit 1 and slit 2, respectively. Usually $D_{\mathrm{p} 2}$ is much smaller than $D_{\mathrm{p} 1}$. Both $D_{\mathrm{p} 1}$ and $D_{\mathrm{p} 2}$ can be derived according to the Fraunhofer diffraction theory as described by Brown and Pomeroy (1989). For a sphere of diameter $d_{\mathrm{p}}$,

$$
\begin{aligned}
D_{\mathrm{p} i}= & \int_{0}^{2 \pi} \int_{\theta_{1}(\varphi)}^{\theta_{2}(\varphi)} I_{0} \frac{d_{\mathrm{p}}^{2}}{16} \alpha^{2}\left|\frac{2 J_{1}(\alpha \sin \theta)}{\alpha \sin \theta}\right|^{2} \\
& \cdot \sin \theta \mathrm{d} \theta \mathrm{d} \varphi(i=1,2),
\end{aligned}
$$

where $\alpha=\pi d_{\mathrm{p}} / \lambda, \lambda$ is the wavelength, and $J_{1}$ is the first order Bessel function of the first kind. The directions of diffracted light, $\theta$ and $\phi$, are defined in the spherical coordinate system fixed on the particle. The interval of integration, and hence $D_{\mathrm{p} i}$, depends on the geometry of the optical system and on the position of the particle.

Equation (2) is rewritten with the effective extinction cross-section, $A_{\mathrm{p} \text {, ext }}$, as

$$
V_{\mathrm{p}} \propto I_{0} A_{\mathrm{p}, \text { ext }} .
$$

In the case of a large particle, diffracted light is confined within a cone of infinitesimal scattering angle and can be detected by the DPD perfectly. This leads to $D_{\mathrm{p} 1}=I_{0} A_{\mathrm{p}}, D_{\mathrm{p} 2}=0$ and $A_{\mathrm{p}, \text { ext }}=A_{\mathrm{p}}$, which is equivalent to the results of the geometrical optics. As the particle size decreases, however, diffracted light diverges and the DPD detects a part of the diffracted energy. Thus $A_{\text {p, ext }}$, exceeds $A_{\mathrm{p}}$.

For a wire of diameter $d_{\mathrm{w}}$, similar relations are written as

$$
\begin{gathered}
V_{\mathrm{w}} \propto 2 I_{0} A_{\mathrm{w}}-D_{\mathrm{w} 1}+D_{\mathrm{w} 2}=I_{0} A_{\mathrm{w}, \mathrm{ext}} \\
D_{\mathrm{w} i}=\int_{x_{1}}^{x_{2}} \int_{\theta_{1}(x)}^{\theta_{2}(x)} I_{0} d_{\mathrm{w}} \frac{\alpha}{\pi}\left|\frac{\sin (\alpha \sin \theta)}{\alpha \sin \theta}\right|^{2} \mathrm{~d} \theta \mathrm{d} x \quad(i=1,2),
\end{gathered}
$$

where the suffix w denotes a wire, $\alpha=\pi d_{\mathrm{w}} / \lambda$, and $A_{\mathrm{w}}$ is the projected area of the wire on slit 1. Both $D_{\mathrm{w} 1}$ and $D_{\mathrm{w} 2}$ are the Fraunhofer diffraction terms, and the integrand of Equation (6) is derived for an infinitely long cylinder according to Van de Hulst (1981). The scattering angle $\theta$ is defined with respect to the cylindrical coordinates fixed

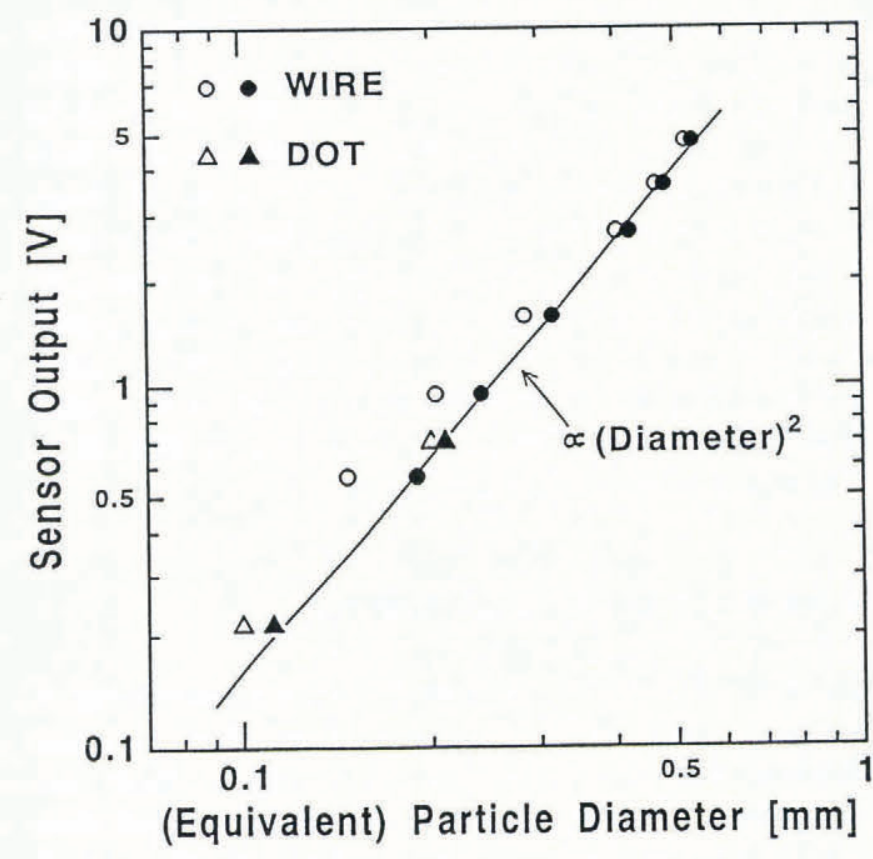

Fig. 4. Calibration results with wires (circles) and dots (triangles). The (equivalent) particle diameter for the open symbol is calculated geometrically, while that for the solid symbol is obtained considering diffraction.

on the wire inclining $45^{\circ}$ to the slit. It should be noted that the interval of integration along the wire is not limited within a slit width, and that the values $A_{\mathrm{w}}, D_{\mathrm{w} 1}, D_{\mathrm{w} 2}$ vary with the position of the projection of the wire.

In order to validate the above treatment, calibration results with wires $\left(d_{\mathrm{w}}=0.024-0.3 \mathrm{~mm}\right)$ are presented in Figure 4. In the figure, sensor outputs are plotted against two equivalent particle diameters, $d_{\mathrm{e}}$ and $d_{\mathrm{e} 0}$, defined by

$$
\begin{gathered}
d_{\mathrm{e}}=2\left(A_{\mathrm{w}} / \pi\right)^{1 / 2,} \\
d_{\mathrm{e} 0}=2\left(A_{\mathrm{w}, \mathrm{ext}} / \pi\right)^{1 / 2},
\end{gathered}
$$

where $A_{\mathrm{w}}$ is calculated geometrically, and $A_{\mathrm{w}, \text { ext }}$ is calculated by Equations (5) and (6). Thus $d_{\mathrm{e}}$ remains in the framework of the geometrical optics, and $d_{\mathrm{e} 0}$ takes account of diffraction. The plotted sensor output $V_{w}$ is the peak output voltage from the preamplifier. Corresponding maximum values of $A_{\mathrm{w}}$ and $A_{\mathrm{w} \text {, ext }}$ are substituted into Equations (7) and (8), which are found from the calculations for various positions of the wire in the middle of the light path.

For reference, another calibration was carried out with dots of two diameters $\left(d_{\mathrm{p}}=0.1 \mathrm{~mm}, 0.2 \mathrm{~mm}\right) .16$ dots of the same diameter are attached to a coaxial circle on a rotating glass disk at regular intervals. The coherent light of the LD interferes due to the inhomogeneity of the glass disk (Kimura, 1991), which causes irregular noise during the rotation. So, the dot-calibration is inherently not practical for the new SPC system.

For comparison with the wire-calibration results, however, about 20 sensor outputs were measured carefully and averaged. As the diffraction theory for a spherical particle can be applied to a dot of the same diameter, averaged sensor outputs are plotted against $d_{\mathrm{p}}$ 
and $d_{\mathrm{p} 0}$ in Figure 4 , where $d_{\mathrm{p}}$ is an actual diameter of the dot and $d_{\mathrm{p} 0}$ is defined by

$$
d_{\mathrm{p} 0}=2\left(A_{\mathrm{p}, \text { ext }} / \pi\right)^{1 / 2}=\left(A_{\mathrm{p}, \text { ext }} / A_{\mathrm{p}}\right)^{1 / 2} d_{\mathrm{p}} .
$$

Here, $A_{\mathrm{p}, \text { ext }}$ is calculated by Equations (2)-(4) for the dot located in the middle of the light path. Equation (9) also takes diffraction into account.

The open symbols in Figure 4, plotted against $d_{\mathrm{e}}$ or $d_{\mathrm{p}}$, show that sensor output is not proportional to the geometrical cross-section (i.e., $d_{\mathrm{e}}^{2}$ or $d_{\mathrm{p}}^{2}$ ). On the other hand, the solid symbols, plotted against $d_{\mathrm{e} 0}$ or $d_{\mathrm{p} 0}$, show that sensor output is in proportion to the effective extinction cross-section (i.e. $d_{\mathrm{e} 0}^{2}$ or $\left.d_{\mathrm{p} 0}^{2}\right)$. Moreover, considering the effects of diffraction by the wire and the dot, both calibration results can be unified.

As a result of the above, Equations (2) and (3) give a relation between an actual particle diameter and sensor output, namely, an actual calibration curve for a blowing snow particle. The remaining unknown constant of proportionality in Equation (2) is determined by fitting Equation (2) to the wire-calibration data plotted against an actual equivalent particle diameter. For the wire of diameter $d_{\mathrm{w}}$, its actual equivalent particle diameter is obtained as a solution $d_{\mathrm{p}}$ of Equation (9) where $d_{\mathrm{e} 0}$ is substituted for $d_{\mathrm{D} 0}$. An example of the actual calibration curve is indicated by the solid line in Figure 5 . The curve extends to a smaller particle-size range which is necessary for mass flux measurement.

\section{FIELD TEST}

Comparative mass flux measurements were conducted with the new SPC system and a snow trap designed by Takeuchi (1980). This trap is a bag made of $150 \mu \mathrm{m}$ mesh plankton net, to which a plastic pipe of $5 \mathrm{~cm}$ length and

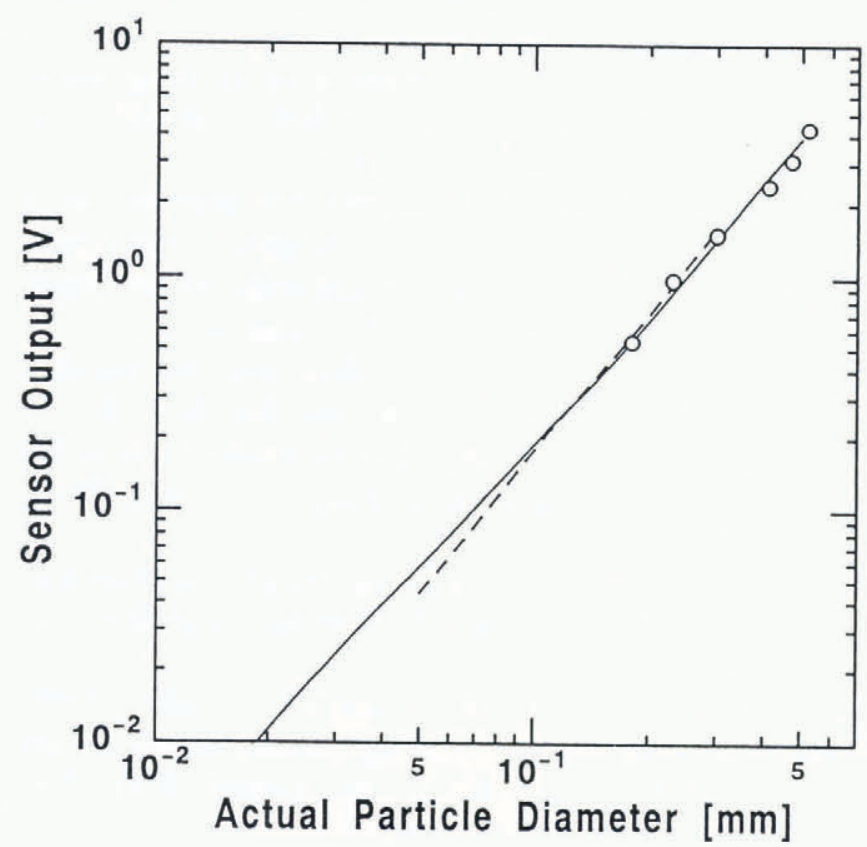

Fig. 5. An example of actual calibration curve (solid line). Open circles are wire-calibration data, which are plotted against the actual equivalent particle diameter defined in the text. The broken line is used in the mass flux calculation.
$2.9 \mathrm{~cm}$ inner diameter is attached. Total length of the bag trap is $26 \mathrm{~cm}$. Abe (1989) determined the collection efficiency of this bag trap to be 0.75 on an average, but the collection efficiency scattered for the wind speed at a level of the trap ranging from 7 to $11 \mathrm{~m} \mathrm{~s}^{-1}$.

Since the functional amplifier of the new SPC system assumes that sensor output is proportional to the square of a particle diameter, the calibration curve should be modified when a mass flux value is calculated from final output voltage. The present study assumes the broken line in Figure 5 for this purpose, which crosses the actual calibration curve at $d_{\mathrm{p}}=130 \mu \mathrm{m}$. The discrepancy between the two curves may cause some error in mass flux measurement.

The measurements were made in January 1992 in the Tsugaru Plain, Aomori Prefecture, Japan. Both the new SPC sensor and the bag trap were mounted at the same height $(0.1,0.2$ and $0.5 \mathrm{~m})$ and opposed to mean wind direction. The distance between the two gauges was less than $50 \mathrm{~cm}$. Wind speed at a height of $4 \mathrm{~m}$ ranged from 6 to $12 \mathrm{~m} \mathrm{~s}^{-1}$.

Results are shown in Figure 6 where each plot corresponds to an average over the period from 1 to 6 min. Although mass fluxes between $10^{-1}$ and $10^{2} \mathrm{~g} \mathrm{~m}^{-2} \mathrm{~s}^{-1}$ were observed, the data with low accuracy are not shown in the figure. The data are scattering around the 1:1 line within a mass flux range of about $10^{0}-10^{2} \mathrm{~g} \mathrm{~m}^{-2} \mathrm{~s}^{-1}$. Most of the mass flux ratios, SPC/bag, are between 0.5 and 2, and their average is 1.2. There is no marked dependence of the ratio on the observation height. Thus the new SPC system was found to give mass fluxes close to those with the bag trap.

The scattering of present data is nearly equal to the results of Schmidt and others (1984) and Schmidt (1986), who compared a large sock trap with the Schmidt's 1977 SPC. Ishimoto and Takeuchi (1987) compared the same

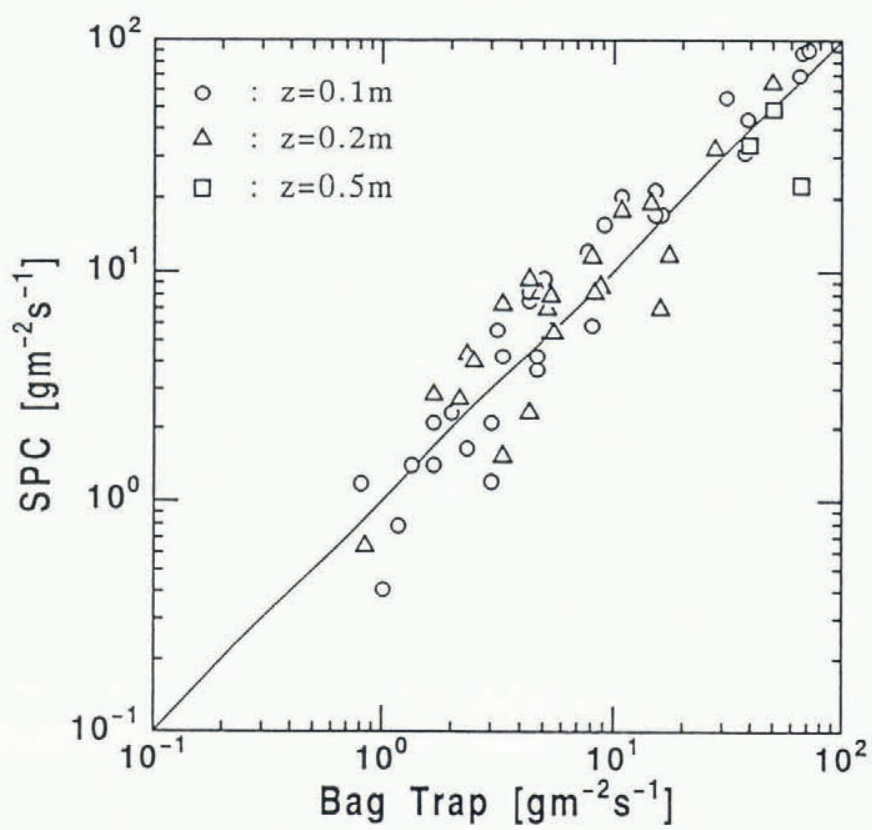

Fig. 6. Comparison of mass flux values. The bag-trap data are corrected with the collection efficiency, 0.75 . 


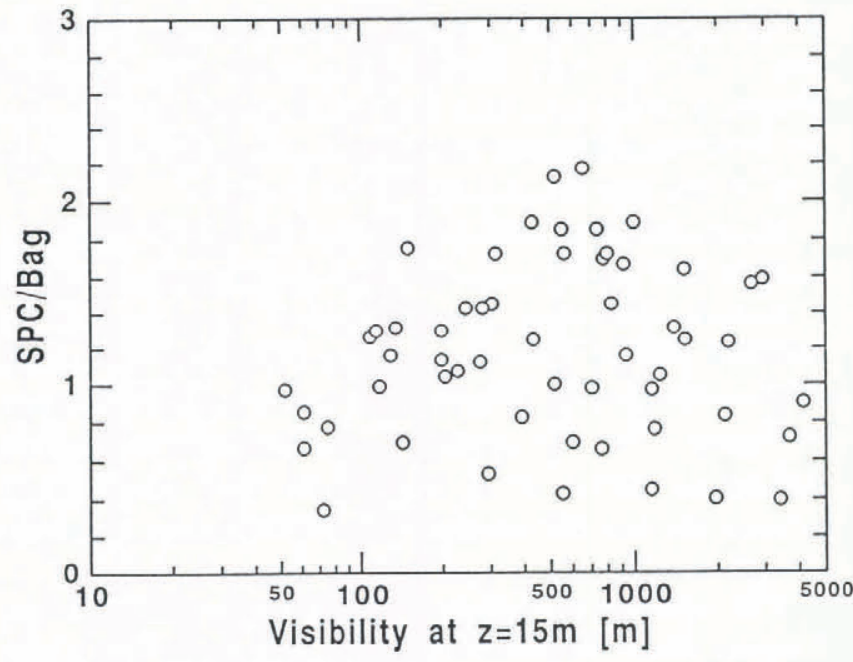

Fig. 7. Relation between visibility at a height of $15 \mathrm{~m}$ and the mass flux ratio.

bag trap as used in the present study with the Schmidt's SPC sensor. They considered only aerodynamic efficiency of the bag trap, and obtained the mean mass flux ratio (SPC/bag) of about 1.4, which is slightly larger than the present result.

Blowing snow accompanied by snowfall contains not only spherical particles but also variously shaped particles including snow crystals and snowflakes, and its size distribution extends to a larger range (Gubler, 1981; Schmidt, 1984). These effects, associated with snowfall, on the SPC measurement are examined from the same data in the following.

Since visibility is well related to snowfall intensity (Fujiyoshi and others, 1983), mass flux ratios are plotted in Figure 7 against the visibility at a height of $15 \mathrm{~m}$, which was observed about $200 \mathrm{~m}$ away from the mass flux observation site. It is expected that the visibility at this level is affected mainly by snowfall. The mass flux ratios are scattering and are not dependent on visibility, namely, on snowfall intensity. This suggests that nonspherical particle shapes and extended size distribution did not influence the mass flux ratios, at least in the comparative measurements. However, the effect of snowfall on the SPC measurement should be investigated under other conditions.

After the comparative measurements, a continuous mass flux observation was carried out. The new SPC system was powered by two $35 \mathrm{~A} \mathrm{~h}$ lead batteries and was confirmed to operate normally for at least nine days.

\section{CONCLUDING REMARKS}

In this study, the new SPC system was confirmed to perform well in the mass flux measurement at a height between $0.1 \mathrm{~m}$ and $0.5 \mathrm{~m}$ for wind speed less than $12 \mathrm{~m} \mathrm{~s}^{-1}$. Comparisons at higher levels and under stronger wind conditions, however, remain as a future subject of investigation.

For use under more severe conditions, well-matched improvement of the optical system and the signal processor may be required.

\section{ACKNOWLEDGEMENTS}

This study was made in the research project "Studies on clarification of drifting snow occurrence and development of disaster prevention technology" by the National Research Institute for Earth Science and Disaster Prevention (NIED). The authors are grateful to Dr M. Higashiura of Shinjo Branch of Snow and Ice Studies, NIED who recorded the bag-trap data in the field. They also thank Dr T. Hayasaka of Tohoku University for his helpful comments on diffraction phenomena.

\section{REFERENCES}

Abe, O. 1989. A collection efficiency of a net-type blowing snow trap meter. Report of the National Research Center for Disaster Prevention 43, 247-254. [In Japanese with English summary.]

Brown, T. and J.W. Pomeroy. 1989. A blowing snow particle detector. Cold Reg. Sci. Technol., 16(2), 167174.

Budd, W.F. 1966. The drifting of nonuniform snow particles. Antarct. Res. Ser., 9, 59-70.

Budd, W.F., W. R.J. Dingle and U. Radok. 1966. The Byrd Snow Drift Project: outline and basic results. Antarct. Res. Ser., 9, 71-134.

Fujiyoshi, Y., G. Wakahama, T. Endoh, S. Irikawa, H. Konishi and M. Takeuchi. 1983. Simultaneous observation of snowfall intensity and visibility in winter at Sapporo. Low Temp. Sci., Ser.A 42, 147-156. [In Japanese with English summary.]

Gubler, H. 1981. An electronic remote snow-drift gauge. f. Glaciol., 27(95), 164-174.

Hulst, H. C. van de. 1981. Light scattering by small particles. New York, Dover.

Ishimoto, K. and M. Takeuchi. 1987. Development of a vehicle-mounted blowing snow monitor. Mon. Rep. Civil Eng. Res. Inst. (Sapporo) 413, 13-17. [In Japanese with English summary.]

Kimura, T. 1991. Measurements of drifting snow particles. 7. Geogr. (Tokyo), 100, 250-263. [In Japanese with English summary.]

Nishimura, H. and N. Maeno. 1983. Size distribution of drifting snow particles at various heights in Antarctica. Proceedings of the 1983 Spring Convention of the Fapan Meteorological Society 43, 111. [In Japanese.]

Schmidt, R.A. 1977. A system that measures blowing snow. U.S. Dep. Agric. For. Serv. Res. Pap. RM-194.

Schmidt, R. A. 1984. Measuring particle size and snowfall intensity in drifting snow. Cold Reg. Sci. Technol., 9(2), 121-129.

Schmidt, R. A. 1986. Transport rate of drifting snow and the mean wind speed profile. Boundary Layer Meteorol., 34(3), 213-241.

Schmidt, R.A., R. Meister and H. Gubler. 1984. Comparison of snow drifting measurements at an Alpine ridge crest. Cold Reg. Sci. Technol., 9(2), 131141.

Takeuchi, M. 1980. Study on visibility in blowing snow. Rep. Civil Eng. Res. Inst. (Sapporo) 74. [In Japanese with English summary.]

The accuracy of references in the text and in this list is the responsibility of the authors, to whom queries should be addressed. 